\title{
COUNTING MULTIPLICITIES IN A HYPERSURFACE OVER NUMBER FIELDS
}

\author{
Hao Wen \& Chunhui Liu
}

\begin{abstract}
We fix a counting function of multiplicities of algebraic points in a projective hypersurface over a number field, and take the sum over all algebraic points of bounded height and fixed degree. An upper bound for the sum with respect to this counting function will be given in terms of the degree of the hypersurface, the dimension of the singular locus, the upper bounds of height, and the degree of the field of definition.
\end{abstract}

\section{Contents}

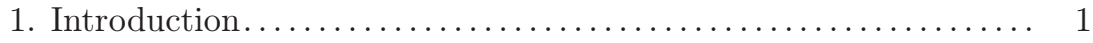

2. Operations over intersection trees.................. 5

3. Counting algebraic points in arithmetic varieties.......... 8

4. Estimate of multiplicities in a hypersurface............. 15

References..................................... 21

\section{Introduction}

In this paper, we consider a problem of counting multiplicities in projective schemes. More precisely, let $k$ be a field, and $X$ be a scheme of finite type over Spec $k$, we are interested in the estimate of the sum

$$
\sum_{\xi \in S(X(\bar{k}))} f\left(\mu_{\xi}(X)\right),
$$

where $S(X(\bar{k}))$ is a subset of $X(\bar{k})$ which satisfies some conditions, and $f(\cdot)$ is a positive function and $\mu_{\xi}(X)$ is the multiplicity of $\xi$ in $X$ defined via the local HilbertSamuel function of $X$ at $\xi$ as follows.

We say that $X$ is a pure dimensional scheme (or $X$ is of pure dimension) if all its irreducible components have the same dimension. Let $X$ be of pure dimension, 
and $\xi \in X$ be a point. Consider the local ring $\mathcal{O}_{X, \xi}$, whose maximal ideal is $\mathfrak{m}_{\xi}$ and residue field is $\kappa(\xi)$. The local Hilbert-Samuel function of $X$ at $\xi$ is given as

$$
H_{\xi}(m)=\operatorname{dim}_{\kappa(\xi)}\left(\mathfrak{m}_{\xi}^{m} / \mathfrak{m}_{\xi}^{m+1}\right)
$$

defined for all $m \in \mathbb{N}^{+}$. Suppose $\operatorname{dim}\left(\mathcal{O}_{X, \xi}\right)=t \geqslant 1$, then there exists a polynomial $P_{\xi}(T)$ of degree $t-1$ such that $H_{\xi}(m)=P_{\xi}(m)$ when $m$ is large enough. In addition, there exists an integer $\mu_{\xi}(X) \geqslant 1$ such that

$$
P_{\xi}(m)=\mu_{\xi}(X) \frac{m^{t-1}}{(t-1) !}+o\left(m^{t-1}\right) .
$$

We define the integer $\mu_{\xi}(X)$ as the multiplicity of the point $\xi$ in $X$. In particular, if the point $\xi$ is regular in $X$, which means that $\mathcal{O}_{X, \xi}$ is a regular local ring, then we have $\mu_{\xi}(X)=1$.

If we take the counting function $f$ as the constant function $f \equiv 1$, then this problem reduces to the classical problem of counting algebraic points on the scheme $X$. There have been many literatures about this problem hitherto. If we take $f$ to be a nontrivial function, and in addition we require $f(1)=0$, then this problem will be a question about the complexity of the singular locus of $X$.

1.1. Known results. - First we consider the case where $X$ is a reduced plane curve of degree $\delta$. In Exercise 5-22 in page 115 of [7], we have

$$
\sum_{\xi \in X} \mu_{\xi}(X)\left(\mu_{\xi}(X)-1\right) \leqslant \delta(\delta-1)
$$

which is obtained by the Bézout's Theorem in the intersection theory. In addition, let $g(X)$ be the genus of $X$, if $X$ is geometrically integral, by Corollary 1 in page 201 of [7], we have

$$
g(X) \leqslant \frac{(\delta-1)(\delta-2)}{2}-\sum_{\xi \in X} \frac{\mu_{\xi}(X)\left(\mu_{\xi}(X)-1\right)}{2}
$$

This inequality is deduced from the Riemann-Roch Theorem of plane curves.

More generally, let $X \hookrightarrow \mathbb{P}_{k}^{n}$ be a projective hypersurface over an algebraically closed field $k$ of characteristic 0 , whose singular locus is of dimension 0 . Through the method of Lefschetz pencil, a direct corollary of [15, Corollaire 4.2.1] gives the inequality

$$
\sum_{\xi \in X} \mu_{\xi}(X)\left(\mu_{\xi}(X)-1\right)^{n-1} \leqslant \delta(\delta-1)^{n-1}
$$

But the condition that the singular locus is of dimension 0 is too restrictive for a general counting problem. In general, the sum in the left hand side of the above inequality depends on the candidate of the base field $k$.

In [17, Théorème 5.1], the second author of this paper obtained a result of this type over finite fields. More precisely, let $n \geqslant 2, \delta \geqslant 1, s \geqslant 0$ be three integers, and 
$\mathbb{F}_{q}$ be the finite field with exact $q$ elements. He proved that the estimate

$$
\sum_{\xi \in X\left(\mathbb{F}_{q}\right)} \mu_{\xi}(X)\left(\mu_{\xi}(X)-1\right)^{n-s-1} \ll_{n} \delta^{n-s} \max \{\delta-1, q\}^{s}
$$

holds uniformly for all reduced hypersurface $X$ of degree $\delta$ of $\mathbb{P}_{K}^{n}$ whose singular locus is of dimension $s$. In the above formula we have used Vinogradov's symbol $\ll$ in its usual sense: let $\Omega$ and $P$ be two sets, and $\widetilde{\Omega}$ be a subset of $\Omega \times P$. Suppose that $f(x, y)$ and $g(x, y)$ are two real-valued functions defined on $\widetilde{\Omega}$, where $x \in \Omega$ and $y \in P$. Then the expression

$$
f(x, y) \ll_{y} g(x, y)
$$

means that there exists a non-negative function $C(\cdot)$ on the set $P$ such that

$$
|f(x, y)| \leqslant C(y)|g(x, y)|
$$

holds for every $(x, y) \in \widetilde{\Omega}$.

Some examples are given in $[\mathbf{1 7}]$ to show that the order of $\delta$ and $\max \{\delta-1, q\}$ in (1.3) are both optimal when $q \geqslant \delta-1$. This estimate is obtained by the technique of intersection tree introduced in $[\mathbf{1 7}, \S 2.1]$ via the intersection theory on projective spaces.

1.2. Principal Result. - In this paper, we consider a sum of the same type as in (1.3) over number fields. More precisely, we take the sum over all the algebraic points, whose fields of definition are of fixed degree over the base field, of bounded height in a hypersurface of a projective space. By the Northcott's property (cf. [14, Theorem B.2.3]), this is a finite set, hence the sum always makes sense. The principle result (Theorem 4.5) is stated as follows:

Theorem 1.1. - Let $K$ be a number field, $n \geqslant 2$ be an integer, and $h(\cdot)$ be the absolute logarithmic height function on $\mathbb{P}_{K}^{n}$. For any closed subscheme $X$ of $\mathbb{P}_{K}^{n}$, any $D \in \mathbb{N}^{+}$, and any $B \geqslant 1$, let

$$
S(X ; D, B)=\{\xi \in X(\bar{K}) \mid[K(\xi): K]=D, \exp ([K(\xi): \mathbb{Q}] h(\xi)) \leqslant B\} .
$$

Let $\delta$ and $s$ be integers such that $\delta \geqslant 1$ and $s \geqslant 0$. Then the inequality

$$
\begin{gathered}
\sum_{\xi \in S(X ; D, B)} \mu_{\xi}(X)\left(\mu_{\xi}(X)-1\right)^{n-s-1} \\
\leqslant \sum_{t=0}^{s} \max _{Z \in \mathcal{Z}_{t}}\left\{\frac{\# S(Z ; D, B)}{\operatorname{deg}(Z)}\right\} \delta(\delta-1)^{n-s+t-1},
\end{gathered}
$$

holds for all reduce hypersurfaces $X$ of degree $\delta$ of $\mathbb{P}_{K}^{n}$ whose singular locus is of dimension $s$, where, for $t \in\{0, \ldots, s\}, \mathcal{Z}_{t}$ is a set of closed subschemes of $X$ of dimension $s-t$, whose construction will be explained in \$4.2.

We keep all the notation in Theorem 1.1. If we want to get an upper bound of the sum

$$
\sum_{\xi \in S(X ; D, B)} \mu_{\xi}(X)\left(\mu_{\xi}(X)-1\right)^{n-s-1}
$$


through Theorem 1.1 for all $X$ satisfying the above conditions, it is important to understand the term

$$
\max _{Z \in \mathcal{Z}_{t}}\left\{\frac{\# S(Z ; D, B)}{\operatorname{deg}(Z)}\right\}
$$

which originates from the classical problem of counting algebraic points, or of counting rational points for the case of $D=1$.

We have the following corollary of Theorem 1.1 for the case of $K=\mathbb{Q}$.

Corollary 1.2 (Corollary 4.7). - With all the notation and conditions of Theorem 1.1. Suppose $K=\mathbb{Q}$, and let $S(X ; B)=S(X ; 1, B)$ for simplicity. Then the estimate

$$
\sum_{\xi \in S(X ; B)} \mu_{\xi}(X)\left(\mu_{\xi}(X)-1\right)^{n-s-1} \ll_{n} \delta^{n-s} \max \{B, \delta-1\}^{s+1}, \quad B \geqslant 1
$$

holds uniformly for all reduce singular hypersurfaces $X$ of degree $\delta$ of $\mathbb{P}_{\mathbb{Q}}^{n}$ whose singular locus is of dimension s.

Moreover, we can construct some examples (for instance, Example 4.8) to show that for all $X$ considered in Theorem 1.1, the exponents of $\delta$ and $\max \{B, \delta-1\}$ in Corollary 1.2 are both optimal when $B \geqslant \delta-1$. We will also explain (Remark 4.6) that the consideration in Theorem 1.1 is necessary.

1.3. Principal Tools. - We shall follow the construction of intersection trees introduced in $[17, \S 2.1]$ to control the multiplicities of singular points. We construct a series of intersections over $\mathbb{P}_{K}^{n}$, and cut $X$ into several irreducible components. The multiplicity of each irreducible component can be bounded by its multiplicity in the intersection trees. Different from techniques used in [17] over a finite field, we work over a number field in this paper, whose cardinality is infinite. Consequently this allows us to work over the original base field directly, and we do not need to take a finite extension of the base field in order to make sure that we can construct useful auxiliary schemes, and then descend it back to the original base field.

Meanwhile, we need to consider the number of rational points and algebraic points of bounded height. Since we require that the constant in the estimate in Corollary 1.2 only depends on $n$, we need a uniform estimate of the number of algebraic points of bounded height in arithmetic varieties, which has a weak dependance on the degrees of varieties.

This paper is organized as follows: in $\S 2$, we introduce the technique of intersection tree in [17]. In $\S 3$, we recall some useful results on counting rational points and algebraic points, and we consider a uniform estimate of rational points of bounded height over $\mathbb{Q}$, which is a generalization of [22, Theorem 1] and [5, Theorem 3.1]. In $\S 4$, we give an upper bound of this multiplicity-counting problem as a function of intersection trees, and we give a uniform upper bound of it via a generalized Schanuel's estimate. 
Acknowledgment. - We would like to thank Dr. Yang Cao and Dr. Enlin Yang for some useful suggestions on some technical details in this paper, and we would like to thank the anonymous referees for their useful comments and suggestions. Chunhui Liu is supported by JSPS KAKENHI Grant Number JP17F17730.

\section{Operations over intersection trees}

In this section, we recall the notion of intersection tree in the settings of graph theory and some useful properties of it. These are introduced in [17]. We fix a base field $k$ throughout this section.

2.1. Preliminaries of intersection theory. - Let $X$ be a projective scheme and $\xi \in X$. In (1.1), we have defined the multiplicity of the point $\xi$ in $X$, noted by $\mu_{\xi}(X)$. In addition, if $M$ is an integral closed subscheme of $X$ whose generic point is $\xi_{M}$, we define the multiplicity of $M$ in $X$ as $\mu_{\xi_{M}}(X)$, noted by $\mu_{M}(X)$ for simplicity.

In the following, we will recall some useful notions and properties of the intersection theory. We will follow the strategy of [24] and [8].

Let $Y$ be a separated regular $k$-scheme of finite type, $r \geqslant 2$ be an integer, and $X_{1}, \ldots, X_{r}$ be pure dimensional closed subschemes of $Y$. We denote by $\mathcal{C}\left(X_{1} \cdot \ldots \cdot X_{r}\right)$ the set of irreducible components of the intersection product $X_{1} \cdot \ldots \cdot X_{r}$. Let $X$ be a pure dimensional closed subscheme of $Y$, we denote by $\mathcal{C}(X)$ the set of irreducible components of $X$. If not specially mentioned, each element of $\mathcal{C}\left(X_{1} \cdot \ldots \cdot X_{r}\right)$ and $\mathcal{C}(X)$ is considered to be an integral closed subscheme of $Y$. Let $M \in \mathcal{C}\left(X_{1} \cdot \ldots \cdot X_{r}\right)$, we denote by

$$
i\left(M ; X_{1} \cdot \ldots \cdot X_{r} ; Y\right)
$$

the intersection multiplicity of the intersection product $X_{1} \cdot \ldots \cdot X_{r}$ at $M$, and we refer readers to $[\mathbf{8}$, Chapter 7 and 8$]$ for its definition.

Let $M \in \mathcal{C}\left(X_{1} \cdot \ldots \cdot X_{r}\right)$, with $r \geqslant 2$. In general, we have (cf. [24, Chap. III, Prop. 17])

$$
\operatorname{dim}(M) \geqslant \operatorname{dim}\left(X_{1}\right)+\cdots+\operatorname{dim}\left(X_{r}\right)-(r-1) \operatorname{dim}(Y) .
$$

If the equality holds and the intersection is not empty, we say that $X_{1}, \ldots, X_{r}$ intersect properly at $M$ in $Y$, and $M$ is a proper component of the intersection product $X_{1} \cdot \ldots \cdot X_{r}$ in $Y$. If $X_{1}, \ldots, X_{r}$ intersect properly at all its irreducible components, we say that $X_{1}, \ldots, X_{r}$ intersect properly.

Bézout's Theorem. - Let $Y$ be a regular projective $k$-scheme and $\mathscr{L}$ be an ample invertible $\mathcal{O}_{Y}$-module. If $X$ is a closed subscheme of $Y$, we denote by $\operatorname{deg}_{\mathscr{L}}(X)$ the degree of $X$ with respect to the invertible $\mathcal{O}_{Y}$-module $\mathscr{L}$, which is defined as $\operatorname{deg}\left(c_{1}(\mathscr{L})^{\operatorname{dim}(X)} \cap[X]\right)$. If $\mathscr{L}$ is the universal bundle $\mathcal{O}_{Y}(1)$, we note the degree by $\operatorname{deg}(X)$ for simplicity.

The Bézout's Theorem is a description of the complexity of a proper intersection in $\mathbb{P}_{k}^{n}$ in terms of degrees with respect to the universal bundles. 
Theorem 2.1 (Bézout's Theorem). — Let $X_{1}, \ldots, X_{r}$ be a family of closed pure dimensional subschemes of $\mathbb{P}_{k}^{n}$, which intersect properly. Then we have

$$
\sum_{Z \in \mathcal{C}\left(X_{1} \cdot \ldots \cdot X_{r}\right)} i\left(Z ; X_{1} \cdot \ldots \cdot X_{r} ; \mathbb{P}_{k}^{n}\right) \operatorname{deg}(Z)=\operatorname{deg}\left(X_{1}\right) \cdots \operatorname{deg}\left(X_{r}\right) .
$$

We refer readers to $[\mathbf{8}$, Proposition 8.4$]$ for more details. See also the equality (1) in page 145 of [8].

2.2. Definition of intersection tree. - Let $Y$ be a regular separated $k$-scheme and $\mathscr{L}$ be an ample invertible $\mathcal{O}_{Y}$-module. Let $\delta \geqslant 1$ be an integer. We call a directed rooted tree $\mathscr{T}$ with labelled vertices and weighted edges an intersection tree of level $\delta$ over $Y$, if it satisfies the following conditions:

1. the vertices of $\mathscr{T}$ are the occurrences of integral closed subschemes of $Y$ (an integral closed subscheme of $Y$ can appear several times in a tree);

2. each vertex $X$ of $\mathscr{T}$ is attached with a label, which is a pure dimensional closed subscheme of $Y$ or empty;

3. a vertex of $\mathscr{T}$ is a leaf if and only if its label is empty;

4. if $X$ is a vertex of $\mathscr{T}$ which is not a leaf, then

- its label $\widetilde{X}$ satisfies the inequality $\operatorname{deg}_{\mathscr{L}}(\tilde{X}) \leqslant \delta$ and the closed subschemes $X$ and $\tilde{X}$ intersect properly in $Y$;

- the children of $X$ are precisely the irreducible components of the intersection product $X \cdot \widetilde{X}$ in $Y$;

- for each child $Z$ of $X$, the edge $\ell$ which links $X$ and $Z$ is attached with a weight $w(\ell)$ which equals the intersection multiplicity $i(Z ; X \cdot \widetilde{X} ; Y)$.

For every fixed intersection tree $\mathscr{T}$, we call any of the complete subtrees of $\mathscr{T}$ an sub-intersection tree, which is necessarily an intersection tree.

Weight of a vertex. - Let $Y$ be a regular separated scheme over Spec $k$, equipped with an ample invertible sheaf $\mathscr{L}$, and $\mathscr{T}$ be an intersection tree over $Y$. For each vertex $X$ of $\mathscr{T}$, we define the weight of $X$ as the product of the weights of all edges in the path which links the root of $\mathscr{T}$ and the vertex $X$, denoted as $w_{\mathscr{T}}(X)$. If $X$ is the root of an intersection tree, we define $w_{\mathscr{T}}(X)=1$ for convenience.

Weight of an integral closed subscheme. - Let $Z$ be an integral closed subscheme of $Y$. We define the weight of $Z$ relative to the tree $\mathscr{T}$ as the sum of the weights of all the occurrences of $Z$ as vertices of $\mathscr{T}$, noted by $W_{\mathscr{T}}(Z)$. If $Z$ does not appear in the tree $\mathscr{T}$ as a vertex, for convenience the weight $W_{\mathscr{T}}(Z)$ is defined to be 0 . Let $Z$ be a vertex in the intersection tree $\mathscr{T}$. When we write $W_{\mathscr{T}}(Z)$, the symbol $Z$ is considered as an integral closed subscheme of $Y$. In other words, we count all the occurrences of the subscheme $Z$ in the intersection tree $\mathscr{T}$.

Example of intersection trees. - We refer the readers to [17, Exemple 3.2] as an example of the notion of intersection tree.

2.3. Estimate of weights of intersection trees. - In order to estimate the weights in intersections trees, we first introduce the following result. 
Theorem 2.2 (Théorème 3.1, [17]). - Suppose that $k$ is a perfect field. Let $\left\{X_{i}\right\}_{i=1}^{r}$ be a family of closed pure dimensional subschemes of $\mathbb{P}_{k}^{n}$ which intersect properly in $\mathbb{P}_{k}^{n}$. For each irreducible component $C \in \mathcal{C}\left(X_{1} \cdot \ldots \cdot X_{r}\right)$, let $\mathscr{T}_{C}$ be an intersection tree whose root is $C$. We consider a vertex $M$ in the intersection trees $\left\{\mathscr{T}_{C}\right\}_{C \in \mathcal{C}\left(X_{1} \cdots \cdot X_{r}\right)}$ which satisfies: for each vertex $Z$ in $\left\{\mathscr{T}_{C}\right\}_{C \in \mathcal{C}\left(X_{1} \cdot \ldots \cdot X_{r}\right)}$, if $M$ is a proper subscheme of $Z$, then there exists an occurrence of $M$ as a descendant of $Z$. Then we have

$$
\sum_{C \in \mathcal{C}\left(X_{1} \cdots \cdot X_{r}\right)} W_{\mathscr{T}_{C}}(M) i\left(C ; X_{1} \cdot \ldots \cdot X_{r} ; \mathbb{P}_{k}^{n}\right) \geqslant \mu_{M}\left(X_{1}\right) \cdots \mu_{M}\left(X_{r}\right),
$$

where $\mu_{M}\left(X_{i}\right)$ is the multiplicity of $M$ in $X_{i}$, defined in (1.1).

Keeping all the notation in Theorem 2.2, we introduce the following notions.

Definition 2.3. - Let $s$ be a non-negative integer. We define $\mathcal{C}_{s}$ as the set of all vertices of depth $s$ in the intersection trees $\mathscr{T}_{C}$, where $C \in \mathcal{C}\left(X_{1} \ldots X_{r}\right)$. In addition, we define $\mathcal{C}_{*}=\bigcup_{s \geqslant 0} \mathcal{C}_{s}$.

We define a subset of $\mathcal{C}_{s}$ for each non-negative integer $s$ as below.

Definition 2.4. - Let $s$ be a non-negative integer. We define $\mathcal{Z}_{s}$ as the subset of $\mathcal{C}_{s}$ of elements $M$ which satisfy the following condition: for every vertex $Z$ of intersection trees $\left\{\mathscr{T}_{C}\right\}_{C \in \mathcal{C}\left(X_{1} \cdots \cdot X_{r}\right)}$, if $M$ is a proper subscheme of $Z$, then there exists a descendant of $Z$ which is an occurrence of $M$. In addition, we define $\mathcal{Z}_{*}=\bigcup_{s \geqslant 0} \mathcal{Z}_{s}$

By definition, we have $\mathcal{Z}_{0}=\mathcal{C}_{0}=\mathcal{C}\left(X_{1} \cdot \ldots \cdot X_{r}\right)$. In fact, Theorem 2.2 is satisfied for every element in $\mathcal{Z}_{*}$.

Definition 2.5. - Let $s$ be a non-negative integer. We denote by $\mathcal{C}_{s}^{\prime}$ (resp. $\mathcal{Z}_{s}^{\prime}, \mathcal{C}_{*}^{\prime}$ and $\mathcal{Z}_{*}^{\prime}$ ) the set of the labels of $\mathcal{C}_{s}$ (resp. $\mathcal{Z}_{s}, \mathcal{C}_{*}$ and $\mathcal{Z}_{*}$ ).

The following proposition is a corollary of Theorem 2.2, which is proved via Theorem 2.1 .

Proposition 2.6 (Proposition 4.6, [17]). - With all the above notation and the conditions in Theorem 2.2, we suppose that all the non-empty elements in $\mathcal{C}_{*}^{\prime}$ have the same dimension. Then we have

$$
\sum_{Z \in \mathcal{Z}_{s}}\left(\prod_{i=1}^{r} \mu_{Z}\left(X_{i}\right)\right) \operatorname{deg}(Z) \leqslant \prod_{i=1}^{r} \operatorname{deg}\left(X_{i}\right) \prod_{j=0}^{s-1} \max _{\widetilde{Z} \in \mathcal{C}_{j}^{\prime}}\{\operatorname{deg}(\widetilde{Z})\},
$$

where we define by convention $\prod_{j=0}^{s-1} \max _{\widetilde{Z} \in \mathcal{C}_{j}^{\prime}}\{\operatorname{deg}(\widetilde{Z})\}=1$ if $s=0$. 


\section{Counting algebraic points in arithmetic varieties}

Let $K$ be a number field. In order to describe the arithmetic complexity of the closed points in $\mathbb{P}_{K}^{n}$, we introduce the following height function.

3.1. Definition of height functions. - Let $K$ be a number field, $\bar{K}$ be an algebraic closure of $K$, and $M_{K}$ be the set of all places of $K$. For every element $x \in K$, we define the absolute value $|x|_{v}=\left|N_{K_{v} / \mathbb{Q}_{v}}(x)\right|_{v}^{\frac{1}{\left[K_{v}: \mathbb{Q} v\right.}}$ for each $v \in M_{K}$, extending the usual absolute values on $\mathbb{Q}_{p}$ or $\mathbb{R}$. In addition, we define $\|\cdot\|_{v}=|\cdot|_{v}^{\left[K_{v}: \mathbb{Q}_{v}\right]}$ for every $v \in M_{K}$.

Definition 3.1. - Let $\xi \in \mathbb{P}_{K}^{n}(\bar{K})$ be a closed point and $K^{\prime}$ be any field such that $\left[K^{\prime}: K\right]<+\infty$ and $\xi \in \mathbb{P}_{K}^{n}\left(K^{\prime}\right)$. We write a $K^{\prime}$-rational homogeneous coordinate of $\xi$ as $\left[x_{0}: \cdots: x_{n}\right]$. We define the absolute logarithmic height of the point $\xi$ as

$$
h(\xi)=\sum_{v \in M_{K^{\prime}}} \frac{1}{\left[K^{\prime}: \mathbb{Q}\right]} \log \left(\max _{0 \leqslant i \leqslant n}\left\{\left\|x_{i}\right\|_{v}\right\}\right),
$$

which is independent of the choice of the projective coordinate by the product formula (cf. [20, Chap. III, Proposition 1.3]).

We can prove that $h(\xi)$ is independent of the choice of the field $K^{\prime}$ (cf. [14, Lemma B.2.1]).

If $\xi$ is an algebraic point of $\mathbb{P}_{K}^{n}$ valued in a number field $K^{\prime}$ containing $K$, we define the relative multiplicative height of the point $\xi$ to be

$$
H_{K^{\prime}}(\xi)=\exp \left(\left[K^{\prime}: \mathbb{Q}\right] h(\xi)\right) .
$$

When considering the closed points of a subscheme $X$ of $\mathbb{P}_{K}^{n}$ with the immersion $\phi: X \hookrightarrow \mathbb{P}_{K}^{n}$, we define the height of $\xi \in X(\bar{K})$ to be

$$
h(\xi):=h(\phi(\xi)) .
$$

We shall use this notation when there is no confusion of the immersion morphism $\phi$.

Let $B \geqslant 1, D \in \mathbb{N}^{+}$, and $X$ be the subscheme of $\mathbb{P}_{K}^{n}$ defined above. We denote by

$$
S(X ; D, B)=\left\{\xi \in X(\bar{K}) \mid[K(\xi): K]=D, H_{K(\xi)}(\xi) \leqslant B\right\},
$$

where $K(\xi)$ is the residue field of $\xi$ in $\mathbb{P}_{K}^{n}$. In particular, we denote by

$$
S(X ; B)=S(X ; 1, B)=\left\{\xi \in X(K) \mid H_{K}(\xi) \leqslant B\right\}
$$

for simplicity. We denote

$$
N(X ; D, B)=\# S(X ; D, B)
$$

and

$$
N(X ; B)=\# S(X ; B) .
$$

By the Northcott's property (cf. [14, Theorem B.2.3]), $N(X ; D, B)$ is finite for every $D \in \mathbb{N}^{+}$and every $B \geqslant 1$.

For the problem of counting rational points or algebraic points, it is essential to understand the functions $N(X ; B)$ and $N(X ; D, B)$ in variables $B$ and $D$. There 
are fruitful results on this topic, and we will introduce some which are useful in the multiplicity-counting problem.

3.2. Schanuel's estimate. - Let $B \geqslant 1, D \in \mathbb{N}^{+}$and $X \hookrightarrow \mathbb{P}_{K}^{n}$ be a projective scheme. With all the notation above, it is natural to consider the density of algebraic points via some properties of $N(X ; D, B)$ and $N(X ; B)$. First we consider the case where $X=\mathbb{P}_{K}^{n}$.

3.2.1. The density of rational points of projective spaces. - For $N\left(\mathbb{P}_{K}^{n} ; B\right)$, we have the following asymptotic estimate

$$
N\left(\mathbb{P}_{K}^{n} ; B\right)=\alpha(K, n) B^{n+1}+o\left(B^{n+1}\right), \quad B \rightarrow+\infty
$$

for all $n \in \mathbb{Z}^{+}$, where the constant $\alpha(K, n)$ is articulated in the paper of S. Schanuel $[22$, Theorem 1].

For the case of $K=\mathbb{Q}$. Let $\xi \in \mathbb{P}_{\mathbb{Q}}^{n}(\mathbb{Q})$, we take the primitive projective coordinate of $\xi$ as $\left[\xi_{0}: \cdots: \xi_{n}\right]$, which means each $\xi_{i} \in \mathbb{Z}$ and $\operatorname{gcd}\left(\xi_{0}, \ldots, \xi_{n}\right)=1$. In this case, we have

$$
H_{\mathbb{Q}}(\xi)=\max _{0 \leqslant i \leqslant n}\left\{\left|\xi_{i}\right|\right\},
$$

where $|\cdot|$ is the usual absolute value. In addition, we have

$$
N\left(\mathbb{P}_{\mathbb{Q}}^{n} ; B\right)=\frac{2^{n}}{\zeta(n+1)} B^{n+1}+o\left(B^{n+1}\right), \quad B \rightarrow+\infty
$$

for all $n \in \mathbb{N}^{+}$, where $\zeta(n)$ is the usual Riemann zeta function. We refer to [5, Theorem 1.2] for a proof, which is simpler than that of [22, Theorem 1]. In this case, we have an explicit uniform estimate of $N\left(\mathbb{P}_{\mathbb{Q}}^{n} ; B\right)$ as following.

Proposition 3.2. - The inequality

$$
N\left(\mathbb{P}_{\mathbb{Q}}^{n} ; B\right) \leqslant 3^{n+1} B^{n+1}
$$

holds for all $B \geqslant 1$ and $n \in \mathbb{N}^{+}$.

Proof. - We consider the set

$$
R\left(\mathbb{A}_{\mathbb{Z}}^{n+1} ; B\right)=\left\{\xi=\left(\xi_{0}, \ldots, \xi_{n}\right) \in \mathbb{A}_{\mathbb{Z}}^{n+1}(\mathbb{Z}) \mid \max _{0 \leqslant i \leqslant n}\{|\xi|\} \leqslant B\right\} .
$$

Because there are at most $2 B+1$ integers whose absolute values are smaller than $B$, we have

$$
\# R\left(\mathbb{A}_{\mathbb{Z}}^{n+1} ; B\right) \leqslant(2 B+1)^{n+1} \leqslant 3^{n+1} B^{n+1} .
$$

In addition, we have $N\left(\mathbb{P}_{\mathbb{Q}}^{n} ; B\right) \leqslant \# R\left(\mathbb{A}_{\mathbb{Z}}^{n+1} ; B\right)$. So we get the result. 
3.2.2. The density of algebraic points of projective spaces. - We have discussed $N\left(\mathbb{P}_{K}^{n} ; B\right)=N\left(\mathbb{P}_{K}^{n} ; 1, B\right)$ above for the case of rational points. For $N\left(\mathbb{P}_{K}^{n} ; D, B\right)$ with arbitrary $D \in \mathbb{N}^{+}$, the situation is very different. Until now, to the authors' knowledge, there is no optimal asymptotic estimate of $N\left(\mathbb{P}_{K}^{n} ; D, B\right)$ for general $n, D$ and $K$. We only have some partial results for these $n, D$ and $K$ satisfying certain conditions.

Let $A(K, n, D)$ be a series of positive constants depending on $n, D \in \mathbb{N}^{+}$and the number field $K$. First we consider the case of $n=1$, in which case $\mathbb{P}_{K}^{n}$ is a projective line. In this case, we have

$$
N\left(\mathbb{P}_{K}^{1} ; D, B\right) \sim A(K, 1, D) B^{D+1}
$$

for all $D \in \mathbb{N}^{+}$and for all number field $K$, see [19] or [16, Théorème 5.1] for a proof, where the constant $A(K, 1, D)$ is explicitly given in the above two references.

Higher dimensional cases are more complicated. Actually, when $n \geqslant 3$, we have

$$
N\left(\mathbb{P}_{K}^{n} ; 2, B\right) \sim A(K, n, 2) B^{n+1}
$$

for all $B \geqslant 1$ and arbitrary number field $K$ in [11, Theorem 1.2.1]), where the constant $A(K, n, 2)$ is given explicitly (loc. cit.). The case of $K=\mathbb{Q}$ is treated in [23].

For the cases of higher extension degrees $D \in \mathbb{N}^{+}$, we have

$$
N\left(\mathbb{P}_{K}^{n} ; D, B\right) \sim A(K, n, D) B^{n+1}
$$

for all $n, D \in \mathbb{N}^{+}$satisfying when $n \geqslant D+2$, or a better estimate

$$
N\left(\mathbb{P}_{K}^{n} ; D, B\right) \ll_{K, D} B^{D+1+\frac{n-1}{D}} \log B, \quad B \rightarrow+\infty
$$

holds uniformly for all $D \geqslant n \geqslant 3$, see [11, Theorem 1.2.2] for the constant $A(K, n, D)$ involved above. For the case of $K=\mathbb{Q}$, this is a theorem in [9].

3.3. A naive estimate for arithmetic varieties. - The aim of this section is to prove the following result.

Theorem 3.3. - Let $n \geqslant 2, \delta \geqslant 1$ and $d \geqslant 1$ be three integers. Then the estimate

$$
S(X ; B) \ll_{n} \delta B^{d+1}, \quad B \geqslant 1
$$

holds uniformly for all pure dimensional closed subscheme $X$ of $\mathbb{P}_{\mathbb{Q}}^{n}$ of dimension $d$ and degree $\delta$.

In order to prove it, we will introduce auxiliary results. First, we introduce the following definition.

Definition 3.4. - Let $k$ be a field, and $X$ be a closed subscheme of $\mathbb{A}_{k}^{n}$. We define the degree of $X$ in $\mathbb{A}_{k}^{n}$ to be the degree of its projective closure in $\mathbb{P}_{k}^{n}$. The degree of $X$ defined above is denoted by $\operatorname{deg}(X)$ if there is no confusion.

By Definition 3.4, we have the following result. 
Lemma 3.5. - Let $k$ be a field, $X \hookrightarrow \mathbb{A}_{k}^{n}$ be a pure dimensional closed subscheme of dimension $d$ with $d \geqslant 1$, and $L$ be a linear subscheme of $\mathbb{A}_{k}^{n}$ which intersects $X$ properly. Then we have

$$
\operatorname{deg}(X) \geqslant \sum_{Z \in \mathcal{C}(X \cap L)} \operatorname{deg}(Z)
$$

where $\operatorname{deg}(\cdot)$ follows Definition 3.4, and $\mathcal{C}(X \cap L)$ is the set of irreducible components of $X \cap L$ considered to be integral closed subschemes of $\mathbb{A}_{k}^{n}$. Moreover, we define $\operatorname{deg}(Z)=1$ by convention if $Z$ is a closed point.

Proof. - Let $\bar{X}$ and $\bar{L}$ be the projective closure of $X$ and $L$ in $\mathbb{P}_{k}^{n}$ respectively, then we have $\operatorname{deg}(X)=\operatorname{deg}(\bar{X})$ and $\operatorname{deg}(L)=\operatorname{deg}(\bar{L})=1$ by Definition 3.4. By the Bézout's Theorem (Theorem 2.1), we have

$$
\operatorname{deg}(\bar{X})=\operatorname{deg}(\bar{X}) \operatorname{deg}(\bar{L})=\sum_{Z \in \mathcal{C}(\bar{X} \cdot \bar{L})} i\left(Z ; \bar{X} \cdot \bar{L} ; \mathbb{P}_{k}^{n}\right) \operatorname{deg}(Z),
$$

where $\mathcal{C}(\bar{X} \cdot \bar{L})$ is the set of irreducible components of the intersection $\bar{X} \cdot \bar{L}$, and $i\left(Z ; \bar{X} \cdot \bar{L} ; \mathbb{P}_{k}^{n}\right)$ is the intersection multiplicity of $\bar{X} \cdot \bar{L}$ at $Z$. For each $Z \in \mathcal{C}(\bar{X} \cdot \bar{L})$, let $a(Z)$ be the restriction of $Z$ in $\mathbb{A}_{k}^{n}$ involved above. By Definition 3.4, we have $\operatorname{deg}(Z)=\operatorname{deg}(a(Z))$ if $a(Z) \neq \emptyset$. So we obtain

$$
\sum_{Z \in \mathcal{C}(\bar{X} \cdot \bar{L})} i\left(Z ; \bar{X} \cdot \bar{L} ; \mathbb{P}_{k}^{n}\right) \operatorname{deg}(Z) \geqslant \sum_{Z \in \mathcal{C}(\bar{X} \cdot \bar{L})} \operatorname{deg}(a(Z))=\sum_{Z \in \mathcal{C}(X \cap L)} \operatorname{deg}(Z),
$$

where we define $\operatorname{deg}(a(Z))=0$ if $a(Z)=\emptyset$ above. The reason is that each intersection multiplicity is larger than or equal to 1 . So we have the result.

We need the following lemma about the definition of Krull dimension of a topological space. We refer the reader its definition at [18, Definition 2.5.1].

Lemma 3.6. - Let $k$ be a field, and $X$ be a non-empty closed irreducible subset of the affine space $\mathbb{A}_{k}^{n}$ whose dimension is $d$, where $d \geqslant 0$. Then $X$ has no proper closed subset of dimension $d$. A proper subset of $X$ means a subset of $X$ which is not equal to $X$ itself.

Proof. - We suppose that $X$ has a proper irreducible closed subset $X^{\prime}$ of dimension $d$. Let

$$
X^{\prime}=X_{0} \supsetneq X_{1} \supsetneq \cdots \supsetneq X_{d}
$$

be a sequence of non-empty irreducible closed subsets of $X^{\prime}$. Then we have the following sequence of non-empty irreducible closed subsets of $X$

$$
X \supsetneq X_{0} \supsetneq X_{1} \supsetneq \cdots \supsetneq X_{d},
$$

which shows that the dimension of $X$ is at least $d+1$. This leads to a contradiction.

Next, we prove a lemma about the intersection of affine schemes. 
Lemma 3.7. - Let $k$ be a field, and $X$ be an irreducible closed subscheme of $\mathbb{A}_{k}^{n}=\operatorname{Spec}\left(k\left[T_{1}, \ldots, T_{n}\right]\right)$, which is of dimension $d$ with $1 \leqslant d \leqslant n-1$. Then there exists an index $\alpha \in\{1, \ldots, n\}$, such that for all $a \in k$, the hyperplane defined by the equation $T_{\alpha}=$ a intersects $X$ properly.

Proof. - For $\alpha \in\{1, \ldots, n\}$ and $a \in k$, we denote by $H\left(T_{\alpha}=a\right)$ the hyperplane defined by the equation $T_{\alpha}=a$. By [24, Chap. III, Prop. 17], we have $\operatorname{dim}(X \cap$ $\left.H\left(T_{\alpha}=a\right)\right) \geqslant d+n-1-n=d-1$ for all $\alpha \in\{1, \ldots, n\}$ and all $a \in k$. By [18, Proposition 2.5.5 (a)], we have

$$
\operatorname{dim}\left(X \cap H\left(T_{\alpha}=a\right)\right) \leqslant \operatorname{dim}(X)=d .
$$

If for each $\alpha \in\{1, \ldots, n\}$, we can find an element $a \in k$ such that $X \cap H\left(T_{\alpha}=a\right)$ is not a proper intersection, which means that we have $\operatorname{dim}\left(X \cap H\left(T_{\alpha}=a\right)\right)=d$ by definition directly.

The set $X \cap H\left(T_{\alpha}=a\right)$ is a closed subset of $X$ and $H\left(T_{\alpha}=a\right)$ by the definition of topological space. By Lemma 3.6, there is no proper closed subset of $X$ whose dimension is $d$ since the scheme $X$ is irreducible and $\operatorname{dim}(X)=d$. From the fact $\operatorname{dim}\left(X \cap H\left(T_{\alpha}=a\right)\right)=d$, we have $X=X \cap H\left(T_{\alpha}=a\right)$. So we obtain $X \subseteq H\left(T_{\alpha}=a\right)$.

From the above hypothesis, for all $\alpha \in\{1, \ldots, n\}$, there exists an element $a \in k$ such that $X \subseteq H\left(T_{\alpha}=a\right)$. For every $\alpha \in\{1, \ldots, n\}$, we choose one of these elements in $k$, noted by $a_{n}$. Then we have $X \subseteq H\left(T_{1}=a_{1}\right) \cap \cdots \cap H\left(T_{n}=a_{n}\right)$. The scheme $H\left(T_{1}=a_{1}\right) \cap \cdots \cap H\left(T_{n}=a_{n}\right)$ is the rational point in $\mathbb{A}_{k}^{n}$ whose affine coordinate is $\left(a_{1}, \ldots, a_{n}\right)$, so we have

$$
X \subseteq\left(a_{0}, \ldots, a_{n}\right) .
$$

This contradicts the fact that $d \geqslant 1$. So we prove the result.

Now we prove a proposition of counting integral points in affine schemes. Before doing this, we introduce a definition of $\mathbb{Z}$-points of a $\mathbb{Q}$-scheme.

Let $\phi: X \hookrightarrow \mathbb{A}_{\mathbb{Q}}^{n}$ be an arbitrary affine subscheme of $\mathbb{A}_{\mathbb{Q}}^{n}$, then we have the following diagram:

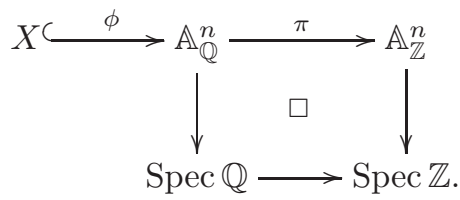

Definition 3.8. - With the above construction, we denote by $X_{\phi}(\mathbb{Z})$ the subset of $X(\mathbb{Q})$ of the $\xi \in X(\mathbb{Q})$ (considered as $\mathbb{Q}$-morphisms from $\operatorname{Spec} \mathbb{Q}$ to $X$ ) whose composition with the canonical immersion morphism $\phi: X \hookrightarrow \mathbb{A}_{\mathbb{Q}}^{n}$ gives a $\mathbb{Z}$-point of $\mathbb{A}_{\mathbb{Z}}^{n}$ having the value in $\mathbb{Q}$ which comes from a $\mathbb{Z}$-point of $\mathbb{A}_{\mathbb{Z}}^{n}$. In other words, we define $X_{\phi}(\mathbb{Z})=X(\mathbb{Q}) \cap \pi^{-1}\left(\mathbb{A}_{\mathbb{Z}}^{n}(\mathbb{Z})\right)$. Instead of $X_{\phi}(\mathbb{Z})$, we denote this set by $X(\mathbb{Z})$ if there is no confusion of the morphism $\phi$.

Proposition 3.9. - For any $B \geqslant 1$, and any subscheme $X$ of $\mathbb{A}_{\mathbb{Q}}^{n+1}$, let

$$
M(X ; B)=\left\{\xi=\left(\xi_{0}, \ldots, \xi_{n}\right) \in X(\mathbb{Z}) \mid \max _{0 \leqslant i \leqslant n}\left\{\left|\xi_{i}\right|\right\} \leqslant B\right\},
$$


where the set $X(\mathbb{Z})$ is defined in Definition 3.8. Let $\delta \geqslant 1, d \geqslant 1$ and $n \geqslant 1$ be three integers, then the estimate

$$
\# M(X ; B) \ll_{n} \delta B^{d}, \quad B \geqslant 1
$$

holds uniformly for all pure dimensional closed subschemes $X$ of $\mathbb{A}_{\mathbb{Q}}^{n+1}$ of dimension $d$ and degree $\delta$.

Proof. - We can suppose that $X$ is irreducible, else we can count it component by component.

We reason by induction on $d$ to prove this lemma. If $d=1$, by Lemma 3.7, there exists an index $\alpha \in\{1, \ldots, n\}$ such that $X$ intersects the hyperplane defined by $T_{\alpha}=a$ properly. Let $H_{a}$ denote this hyperplane. Then we have

$$
M(X ; B)=\bigcup_{\substack{a \in \mathbb{Z} \\|a| \leqslant B}} M\left(X \cap H_{a} ; B\right), \quad B \geqslant 1 .
$$

From Lemma 3.5, each set $M\left(X \cap H_{a} ; B\right)$ contains at most $\delta$ closed points. By the fact that there are at most $2 B+1$ integers whose absolute values are smaller than $B$, we get

$$
M(X ; B) \leqslant \delta(2 B+1), \quad B \geqslant 1,
$$

which proves the case of $d=1$.

Next, we suppose that $d \geqslant 2$. In this case, by Lemma 3.7, we can find an index $\alpha \in\{0, \ldots, n\}$ such that $X$ intersects the hyperplane defined by $T_{\alpha}=a$ properly for any $a \in \mathbb{Q}$. Let $H_{a}$ denote this hyperplane. Then we have

$$
M(X ; B)=\bigcup_{\substack{a \in \mathbb{Z} \\|a| \leqslant B}} M\left(X \cap H_{a} ; B\right), \quad B \geqslant 1 .
$$

For every $a \in \mathbb{Z}$ above, the scheme $X \cap H_{a}$ has dimension at most $d-1$. By Lemma 3.5 , we have the inequality

$$
\delta=\operatorname{deg}(X) \geqslant \sum_{Z \in \mathcal{C}\left(X \cap H_{a}\right)} \operatorname{deg}(Z),
$$

where $\mathcal{C}\left(X \cap H_{a}\right)$ is the set of irreducible components of $X \cap H_{a}$.

By the induction hypothesis, we have

$$
\# M(Z ; B) \ll_{n} \operatorname{deg}(Z) B^{d-1}, \quad B \geqslant 1
$$

for all $Z \in \mathcal{C}\left(X \cap H_{a}\right)$. So we have

$$
\# M\left(X \cap H_{a} ; B\right) \ll_{n} \delta B^{d-1}, \quad B \geqslant 1 .
$$

On the other hand, by the relation (3.8), we have

$$
\# M(X ; B) \leqslant \sum_{\substack{a \in \mathbb{Z} \\|a| \leqslant B}} \# M\left(X \cap H_{a} ; B\right), \quad B \geqslant 1 .
$$

There are at most $2 B+1$ integers whose absolute values are smaller than $B$. So we have the result from the induction hypothesis. 
With the help of Lemma 3.9, we can prove Theorem 3.3. The main idea comes from the proof of [5, Theorem 3.1].

Proof of Theorem 3.3. — Let $\hat{X}$ be the affine cone of $X$ in $\mathbb{A}_{\mathbb{Q}}^{n+1}$. Then we have

$$
N(X ; B) \leqslant \# M(\hat{X} ; B), \quad B \geqslant 1,
$$

where the set $M(\hat{X} ; B)$ follows the notation in the statement of Proposition 3.9. By [12, Chap. I, Exercise 2.10], the scheme $\hat{X}$ is of pure dimension, whose dimension is $d+1$.

Let $\bar{X}$ be the projective closure of $\hat{X}$ in $\mathbb{P}_{\mathbb{Q}}^{n+1}$. Suppose $X=\operatorname{Proj}\left(\mathbb{Q}\left[T_{0}, \ldots, T_{n}\right] / \mathfrak{a}_{X}\right)$ and $\bar{X}=\operatorname{Proj}\left(\mathbb{Q}\left[T_{0}, \ldots, T_{n+1}\right] / \mathfrak{a}_{\bar{X}}\right)$. By $[\mathbf{1 0},(8.3 .1 .1),(8.3 .1 .2)]$, we have

$$
\mathbb{Q}\left[T_{0}, \ldots, T_{n+1}\right] / \mathfrak{a}_{\bar{X}}=\left(\mathbb{Q}\left[T_{0}, \ldots, T_{n}\right] / \mathfrak{a}_{X}\right) \otimes_{\mathbb{Q}} \mathbb{Q}\left[T_{n+1}\right]
$$

as $\mathbb{Q}$-vector spaces.

By [6, Corollary 1.1.13], we obtain that the Hilbert function of $\bar{X}$ is the convolution of the Hilbert function of $X$ and that of $\operatorname{Proj}\left(\mathbb{Q}\left[T_{n+1}\right]\right)$. By [6, Lemma 1.1.12], we get $\operatorname{deg}(\hat{X})=\operatorname{deg}(\bar{X})=\operatorname{deg}(X)=\delta$ which follows Definition 3.4. So we get the result from Proposition 3.9 .

3.4. Varieties of degree larger than 1. - Let $X \hookrightarrow \mathbb{P}_{K}^{n}$ be an integral closed projective scheme over the number field $K$, whose dimension is $d$ and degree is $\delta$. In Theorem 3.3, we give the optimal response to the case of $\delta=1$. Actually, the scheme $X$ is isomorphic to $\mathbb{P}_{K}^{d}$ in this case.

We consider the cases which the degree is greater than or equal to 2. In [13, Conjecture 2], D. R. Heath-Brown conjectured that, if $d, \delta$ and $n$ are integers such that $d \geqslant 2, \delta \geqslant 3, n \geqslant 4$, and $B \geqslant 1$ be a real number. Then for any $\epsilon>0$, the estimate

$$
N(X ; B) \ll_{n, \epsilon, K} B^{d+\epsilon}
$$

or a weaker one

$$
N(X ; B) \ll_{n, \epsilon, K, \delta} B^{d+\epsilon}
$$

hold uniformly for every integral closed subscheme $X$ of $\mathbb{P}_{K}^{n}$ of degree $\delta$ and dimension $d$. He has also given a proof for the case of $\delta=2$. In order to solve this conjecture, T. Browning, D. R. Heath-Brown and P. Salberger have published several papers on this topic, see $[\mathbf{1}, \mathbf{2}, \mathbf{3}, \mathbf{4}, \mathbf{2 1}]$ for their works on this subject and [5, Chapter 3] for a survey. In their former work, they imposed some technical conditions of $X$. For example, in [21, Theorem 0.1], P. Salberger proved [13, Conjecture 2] when $X$ contains finitely many linear locus of codimension 1 and $\delta \geqslant 4$.

When we consider the conjecture (3.10), another important issue is to consider the order of $\delta$ in this estimate. This estimate will be useful for this multiplicity-counting problem, see $\S 4.3$. 


\section{Estimate of multiplicities in a hypersurface}

In order to study the multiplicities in a projective hypersurface, first we introduce some facts about the multiplicity of a point in a hypersurface.

4.1. Multiplicity in a section of hypersurface. - Let $k$ be an arbitrary field, and $f \in k\left[T_{0}, \ldots, T_{n}\right]$ be a non-zero homogeneous polynomial of degree $\delta$. We consider the scheme

$$
X=V(f)=\operatorname{Proj}\left(k\left[T_{0}, \ldots, T_{n}\right] /(f)\right) .
$$

In fact, the scheme $X$ is a pure dimensional closed subscheme of $\mathbb{P}_{k}^{n}$ and it is a hypersurface in it. We can prove that $X$ is of degree $\delta$ (cf. [12, Proposition 7.6, Chap. I]).

Let $\alpha \in[0, \delta] \cap \mathbb{N}$. We denote by $\mathcal{T}^{\alpha}(f)$ be $k$-vector space spanned by all the partial derivatives of $f$ of order $\alpha$ which are of the following form

$$
\frac{\partial^{|I|} f}{\partial T^{I}}=\frac{\partial^{i_{0}+\cdots+i_{n}} f}{\partial T_{0}^{i_{0}} \cdots \partial T_{n}^{i_{n}}}
$$

for $I=\left(i_{0}, \cdots, i_{n}\right) \in \mathbb{N}^{n+1}$ with $|I|=i_{0}+\cdots+i_{n}=\alpha$. These elements are homogeneous polynomials of degree $\delta-\alpha$.

The following proposition is an explicit criterion in determining the multiplicity of a point in a hypersurface.

Proposition 4.1 (Corollaire 5.4, [17]). - Let $k$ be a arbitrary field of characteristic $0, X \hookrightarrow \mathbb{P}_{k}^{n}$ be a hypersurface defined by an arbitrary non-zero homogeneous polynomial $f$ of degree $\delta, \eta \in X$ be an arbitrary point, and $\alpha$ be an arbitrary integer in $\left[0, \mu_{\eta}(X)-1\right]$. Then for every non-zero $g \in \mathcal{T}^{\alpha}(f)$, the point $\eta$ is contained in the hypersurface $X^{\prime}$ defined by $g$. On the contrary, there exists a non-zero element $g^{\prime} \in \mathcal{T}^{\mu_{\eta}(X)}(f)$, such that $\eta$ is not contained in the hypersurface defined by $g^{\prime}$.

In particular, if $\mu_{\eta}(X)>1$, then for each non-zero $g \in \mathcal{T}^{1}(f), \eta$ lies in the hypersurface defined by $g$. An immediate consequence of this proposition is that for each hypersurface $X^{\prime}$ mentioned in Proposition 4.1, we have $\mu_{\eta}\left(X^{\prime}\right) \geqslant \mu_{\eta}(X)-\alpha$, $\forall \alpha \in\left[0, \mu_{\eta}(X)-1\right]$

4.2. Construction of intersection trees. - By virtue of Proposition 4.1, we can construct a family of intersection trees to solve the multiplicity-counting problem. First, we introduce the following proposition to construct the roots of these intersection trees.

Proposition 4.2 (Lemme 5.8, [17]). - Let $K$ be an arbitrary number field, $f$ be an arbitrary non-zero homogeneous polynomial of degree $\delta$ in $K\left[T_{0}, \ldots, T_{n}\right]$, and $s \in[0, n-2] \cap \mathbb{N}$. We denote by $V(f)$ the projective hypersurface defined by $f$. If the singular locus of $V(f)$ is of dimension $s$, then there exists a family of directional derivatives $g_{1}, \ldots, g_{n-s-1} \in \mathcal{T}^{1}(f)$ of $f$, such that the equality

$$
\operatorname{dim}\left(V(f) \cap V\left(g_{1}\right) \cap \cdots \cap V\left(g_{n-s-1}\right)\right)=s
$$


is verified. In the other words, $V(f) \cap V\left(g_{1}\right) \cap \cdots \cap V\left(g_{n-s-1}\right)$ is a complete intersection.

Let $K$ and $f$ be the same as in Proposition 4.2. We denote by

$$
X=\operatorname{Proj}\left(K\left[T_{0}, \ldots, T_{n}\right] /(f)\right)
$$

in the following argumentation. We denote by $X^{\text {reg }}$ the regular locus of $X$, and by $X^{\text {sing }}$ the singular locus of $X$. Following the notation and conditions in Proposition 4.2 , we denote by $X_{i}$ the hypersurface $V\left(g_{i}\right)$ for simplicity below, where $i=1, \ldots, n-$ $s-1$. By the Jacobian criterion (cf. [18, Theorem 4.2.19]), we have $X^{\text {sing }} \subseteq$ $X \cap X_{1} \cap \cdots \cap X_{n-s-1}$.

For every integral closed subscheme $M$ of $X$, we denote by $M^{(a)}$ the locus of the points $\xi$ in $M$ whose multiplicities $\mu_{\xi}(X)$ are equal to $\mu_{M}(X)$, and by $M^{(b)}$ the locus of the points $\xi$ in $M$ whose multiplicities $\mu_{\xi}(X)$ are greater than or equal to $\mu_{M}(X)+1$. In addition, let $L / K$ be an extension of fields, and we denote by $M^{(a)}(L)$ (resp. $M^{(b)}(L)$ ) the set of $L$-rational points of $M^{(a)}$ (resp. $\left.M^{(b)}\right)$. With this notation, we have $M(L)=M^{(a)}(L) \bigsqcup M^{(b)}(L)$.

By Proposition 4.1, we obtain that $M^{(a)}$ is dense in $M$ since $M^{\text {reg }}$ is dense in $M$ and all of them have multiplicity $\mu_{M}(X)$. The dimension of $M^{(b)}$ is equal to or smaller than $\operatorname{dim}(M)-1$.

Next, we construct a family of intersection trees $\left\{\mathscr{T}_{C}\right\}$, where $C \in \mathcal{C}\left(X \cdot X_{1}, \cdot \ldots\right.$. $\left.X_{n-s-1}\right)$. The root of the intersection tree $\mathscr{T}_{C}$ is $C$.

In order to construct those vertices whose depth are equal to or larger than 1 , let $M$ be a vertex which is already constructed in these intersection trees $\left\{\mathscr{T}_{C}\right\}$. We regard $M$ as an integral closed subscheme of $X$. Next, we consider the set $M(\bar{K})$. If $M^{(b)}(\bar{K})=\emptyset$, then the vertex $M$ is a leaf in one of these intersection trees.

If $M^{(b)}(\bar{K}) \neq \emptyset$, let $\xi \in M^{(b)}(\bar{K})$, then we have $\mu_{M}(X)<\mu_{\xi}(X) \leqslant \delta$. By Proposition 4.1, for a fixed point $\xi^{\prime} \in M^{(a)}(\bar{K})$, we can find some $h \in \mathcal{T}^{\delta-\mu_{M}(X)}(f)$, such that the hypersurface defined by $h$ does not contain $\xi^{\prime}$. In this case, the hypersurface $V(h)$ does not contain the generic point of $M$. By comparing the dimensions of $V(h)$ and $M$, we obtain that $V(h)$ intersects $M$ properly. Of course we have $\operatorname{deg}(h) \leqslant \delta-1$. In this case, we define $V(h)$ as the label $\widetilde{M}$ of $M$. The children of $M$ hence are the irreducible components of the intersection $M \cdot \widetilde{M}$. The weights of the edges are the intersection multiplicities respectively.

For the construction that follows, all the mentioned labels are of dimension $n-1$, hence all the vertices in $\mathcal{C}_{w}$ are of dimension $s-w$, where $1 \leqslant w \leqslant s$ is an integer. The construction terminates in finite steps.

The following lemma is a property of the set $\mathcal{Z}_{*}$ (see Definition 2.4), which will be useful in the proof of Theorem 4.5.

Lemma 4.3 (Lemme 5.9, [17]). - With all the notation and construction above, for every $\xi \in X^{\operatorname{sing}}(\bar{K})$, there exists at least one $Z \in \mathcal{Z}_{*}$ such that $\xi \in Z^{(a)}(\bar{K})$.

Remark 4.4. - In the original proof of Lemma 4.3 in [17, Lemme 5.9], we work over a finite field. In fact this result remains true for the case of a number field, since the proof of [17, Lemme 5.9] only uses the assumption that the base field is perfect. 
4.3. Counting Multiplicities. - With the construction above, we are going to prove the following result.

Theorem 4.5. - Let $K$ be an arbitrary number field, $n \geqslant 2, \delta \geqslant 1$ and $s \geqslant 0$ be three integers. Then the inequality

$$
\begin{gathered}
\sum_{\xi \in S(X ; D, B)} \mu_{\xi}(X)\left(\mu_{\xi}(X)-1\right)^{n-s-1} \\
\leqslant \sum_{t=0}^{s} \max _{Z \in \mathcal{Z}_{t}}\left\{\frac{N(Z ; D, B)}{\operatorname{deg}(Z)}\right\} \delta(\delta-1)^{n-s+t-1},
\end{gathered}
$$

is verified for all reduced hypersurfaces $X$ of $\mathbb{P}_{K}^{n}$ of degree $\delta$, whose dimension of singular locus is $s$. In this inequality, $S(X ; D, B)$ is defined in $(3.1), N(X ; D, B)$ is defined in (3.3), and $\mathcal{Z}_{t}$ is defined in Definition 2.4 following the construction in \$4.2. If $\mathcal{Z}_{t}=\emptyset$ for some $0 \leqslant t \leqslant s$, we define $\max _{Z \in \mathcal{Z}_{t}}\left\{\frac{N(Z ; D, B)}{\operatorname{deg}(Z)}\right\}=0$ by convention.

Proof. - Suppose that a family of intersection trees $\left\{\mathscr{T}_{C}\right\}$ whose roots are the elements in $\mathcal{C}\left(X \cdot X_{1} \cdot \ldots \cdot X_{n-s-1}\right)$ has already been constructed via procedures introduced in $\S 4.2$.

First, we have

$$
\sum_{\xi \in S(X ; D, B)} \mu_{\xi}(X)\left(\mu_{\xi}(X)-1\right)^{n-s-1}=\sum_{\xi \in S\left(X^{\sin ;} ; D, B\right)} \mu_{\xi}(X)\left(\mu_{\xi}(X)-1\right)^{n-s-1},
$$

since for every $\xi \in X^{\text {reg }}$, we always have $\mu_{\xi}(X)=1$.

By Lemma 4.3 , for each $\xi \in X^{\operatorname{sing}}(\bar{K})$, we can find a $Z \in \mathcal{Z}_{*}$ such that $\xi \in Z^{(a)}(\bar{K})$. So we have

$$
\begin{aligned}
& \sum_{\xi \in S\left(X^{\operatorname{sing} ; D, B)}\right.} \mu_{\xi}(X)\left(\mu_{\xi}(X)-1\right)^{n-s-1} \\
& \leqslant \sum_{t=0}^{s} \sum_{Z \in \mathcal{Z}_{t}} \sum_{\xi \in S\left(Z^{(a)} ; D, B\right)} \mu_{\xi}(X)\left(\mu_{\xi}(X)-1\right)^{n-s-1} .
\end{aligned}
$$

By Proposition 4.1, for every $Z \in \mathcal{Z}_{*}$, we have the inequality

$$
\mu_{Z}(X)-1 \leqslant \mu_{Z}\left(X_{i}\right)
$$

for all $i=1, \ldots, n-s-1$. So we get the inequality

$$
\mu_{Z}(X)\left(\mu_{Z}(X)-1\right)^{n-s-1} \leqslant \mu_{Z}(X) \mu_{Z}\left(X_{1}\right) \cdots \mu_{Z}\left(X_{n-s-1}\right) .
$$

By Proposition 2.6 and the inequality (4.3), we have

$$
\begin{aligned}
& \sum_{Z \in \mathcal{Z}_{t}} \mu_{Z}(X)\left(\mu_{Z}(X)-1\right)^{n-s-1} \operatorname{deg}(Z) \\
\leqslant & \sum_{Z \in \mathcal{Z}_{t}} \mu_{Z}(X) \mu_{Z}\left(X_{1}\right) \cdots \mu_{Z}\left(X_{n-s-1}\right) \operatorname{deg}(Z) \\
\leqslant & \operatorname{deg}(X) \prod_{i=1}^{n-s-1} \operatorname{deg}\left(X_{i}\right) \prod_{j=0}^{t-1} \max _{\widetilde{Z} \in \mathcal{C}_{t}^{\prime}}\{\operatorname{deg}(\widetilde{Z})\} \leqslant \delta(\delta-1)^{n-s+t-1},
\end{aligned}
$$


for each $t=0, \ldots, s$, since all the labels in $\mathcal{C}_{*}^{\prime}$ are of degree equal to or smaller than $\delta-1$.

Combine the inequalities (4.2) and (4.4), we obtain that

$$
\begin{aligned}
& \sum_{t=0}^{s} \sum_{Z \in \mathcal{Z}_{t}} \sum_{\xi \in S\left(Z^{(a)} ; D, B\right)} \mu_{\xi}(X)\left(\mu_{\xi}(X)-1\right)^{n-s-1} \\
= & \sum_{t=0}^{s} \sum_{Z \in \mathcal{Z}_{t}} \mu_{Z}(X)\left(\mu_{Z}(X)-1\right)^{n-s-1} N\left(Z^{(a)} ; D, B\right) \\
\leqslant & \sum_{t=0}^{s} \sum_{Z \in \mathcal{Z}_{t}} \mu_{Z}(X)\left(\mu_{Z}(X)-1\right)^{n-s-1} N(Z ; D, B) \\
\leqslant & \sum_{t=0}^{s} \max _{Z \in \mathcal{Z}_{t}}\left\{\frac{N(Z ; D, B)}{\operatorname{deg}(Z)}\right\}\left(\sum_{Z \in \mathcal{Z}_{t}} \mu_{Z}(X)\left(\mu_{Z}(X)-1\right)^{n-s-1} \operatorname{deg}(Z)\right) \\
\leqslant & \sum_{t=0}^{s} \max _{Z \in \mathcal{Z}_{t}}\left\{\frac{N(Z ; D, B)}{\operatorname{deg}(Z)}\right\} \delta(\delta-1)^{n-s+t-1} .
\end{aligned}
$$

By the inequalities (4.1), (4.2) and (4.5), we prove the result.

Remark 4.6. - We keep all the notation and conditions in Theorem 4.5. Let $f: \mathbb{N}^{+} \rightarrow \mathbb{N}$ be an increasing function which is asymptotic to a polynomial whose degree is smaller than $n-s-1$, and it satisfies $f(1)=0$. Then there exists a constant $C_{f}$ depending only on the function $f$, such that $f(x) \leqslant C_{f} \cdot x(x-1)^{n-s-1}$ for all $x \geqslant 1$. Then the inequality

$$
\sum_{\xi \in S(X ; D, B)} f\left(\mu_{\xi}(X)\right) \leqslant C_{f} \sum_{\xi \in S(X ; D, B)} \mu_{\xi}(X)\left(\mu_{\xi}(X)-1\right)^{n-s-1}
$$

is verified for all funtions $f$ satisfying the above conditions, where $D \in \mathbb{N}^{+}$and $B \geqslant 1$. If we do not care about the constant depending on the above function $f(x)$, for this kind of counting multiplicities problem, it is enough to consider the counting function $f(x)=x(x-1)^{n-s-1}$ only, which is considered in Theorem 4.5.

If we consider another increasing counting function $g: \mathbb{N}^{+} \rightarrow \mathbb{N}$ which is asymptotic to a polynomial whose degree is smaller than $n-s-1$, and we do not suppose the condition $g(1)=0$ any longer. Then we have

$$
\begin{aligned}
\sum_{\xi \in S(X ; D, B)} g\left(\mu_{\xi}(X)\right) & =g(1) N\left(X^{\mathrm{reg}} ; D, B\right)+\sum_{\xi \in S(X ; D, B)}\left(g\left(\mu_{\xi}(X)\right)-g(1)\right) \\
& \leqslant g(1) N(X ; D, B)+\sum_{\xi \in S(X ; D, B)}\left(g\left(\mu_{\xi}(X)\right)-g(1)\right) .
\end{aligned}
$$

We consider the sum

$$
\sum_{\xi \in S(X ; D, B)}\left(g\left(\mu_{\xi}(X)\right)-g(1)\right)
$$


by the above discussion, and consider the term $g(1) N(X ; D, B)$ as the classical problem of counting algebraic points or rational points (when $D=1$ ). By the fact that $X^{\text {reg }}$ and $X$ are birational equivalent, this estimate is appropriate.

4.4. The case of rational points. - For the sum considered in Theorem 4.5, now we consider the case of counting multiplicities of rational points. If we want a uniform upper bound of it, we have the following result, which is a corollary of Theorem 4.5 combined with the generalized Schanuel's estimate (Theorem 3.3).

Corollary 4.7. - Let $n \geqslant 2, \delta \geqslant 1$ and $s \geqslant 0$ be three integers. Then the estimate

$$
\sum_{\xi \in S(X ; B)} \mu_{\xi}(X)\left(\mu_{\xi}(X)-1\right)^{n-s-1} \ll_{n} \delta^{n-s} \max \{\delta-1, B\}^{s+1}, \quad B \geqslant 1
$$

holds uniformly for reduced hypersurfaces $X$ of $\mathbb{P}_{\mathbb{Q}}^{n}$ of degree $\delta$ whose singular locus is of dimension $s$, where $S(X ; B)$ is defined in $(3.2)$.

Proof. - By the argumentations in Theorem 3.3, we obtain the estimate

$$
\frac{N(Z ; B)}{\operatorname{deg}(Z)} \ll_{n} B^{\operatorname{dim}(Z)+1}, \quad B \geqslant 1
$$

holds uniformly for $Z \in \mathcal{Z}_{t}$, where $t=0, \ldots, s$ following the construction in $\S 4.2$. Combine the above inequality with the estimate in Theorem 4.5 and the fact that $\operatorname{dim}(Z)<n$ and $s<n$, we obtain the result.

Example 4.8. - Let $X^{\prime} \hookrightarrow \mathbb{P}_{\mathbb{Q}}^{2}$ be a reduced plane curve of degree $\delta$, which is defined by the homogeneous equation $f\left(T_{0}, T_{1}, T_{2}\right)=0$. Suppose that $X^{\prime}$ has a $\mathbb{Q}$ rational point of multiplicity $\delta$. We consider $f$ to be a homogeneous polynomial of degree $\delta$ in $\mathbb{Q}\left[T_{0}, \ldots, T_{n}\right]$ for an integer $n \geqslant 3$. Then $f$ defines a hypersurface in $\mathbb{P}_{\mathbb{Q}}^{n}$, denoted by $X$. Without loss of generality, we suppose that $[1: 0: 0]$ is the projective coordinate of this singular point of $X^{\prime}$. Then we have

$$
\begin{aligned}
X^{\operatorname{sing}}(\mathbb{Q})= & \left\{\left[x_{0}: \cdots: x_{n}\right] \in \mathbb{P}_{\mathbb{Q}}^{n}(\mathbb{Q}) \mid x_{0}=1, x_{1}=0, x_{2}=0\right\} \cup \\
& \left\{\left[x_{0}: \cdots: x_{n}\right] \in \mathbb{P}_{\mathbb{Q}}^{n}(\mathbb{Q}) \mid x_{0}=x_{1}=x_{2}=0\right\},
\end{aligned}
$$

where all singular points of $X$ are of multiplicity $\delta$, and $X^{\text {sing }}$ is considered to be a reduced closed subscheme of $\mathbb{P}_{\mathbb{Q}}^{n}$. By the equality (3.6), we have

$$
N\left(X^{\text {sing }} ; B\right)=N\left(\mathbb{P}_{\mathbb{Q}}^{n-2} ; B\right)=\frac{2^{n-2}}{\zeta(n-1)} B^{n-1}+o\left(B^{n-1}\right), \quad B \rightarrow+\infty .
$$

Then we have the following asymptotic estimate

$$
\sum_{\xi \in S(X ; B)} \mu_{\xi}(X)\left(\mu_{\xi}(X)-1\right)=\delta(\delta-1) N\left(X^{\text {sing }} ; B\right)=O_{n}\left(\delta^{2} B^{n-1}\right)
$$

for each hypersurface $X$ satisfying the above conditions.

From this example, the order of $\delta$ in Corollary 4.7 is optimal when $\operatorname{dim}\left(X^{\operatorname{sing}}\right)=$ $n-2$ and $n \geqslant 3$. More generally, if $X^{\text {sing }}$ contains a linear locus of multiplicity $\delta$ in $X$, we can get the maximal order of $\delta$ and $\max \{B, \delta-1\}$ in the estimate of Corollary 4.7 when $B \geqslant \delta-1$. 
Let $K$ be a number field, and $X \hookrightarrow \mathbb{P}_{K}^{n}$ be a fixed hypersurface of degree $\delta$. To attack this kind of counting multiplicities problem by applying Theorem 4.5, the key point is the uniform estimate of the term

$$
\max _{Z \in \mathcal{Z}_{t}}\left\{\frac{N(Z ; D, B)}{\operatorname{deg}(Z)}\right\}
$$

for all possible $Z \in \mathcal{Z}_{t}(t=1, \ldots, s)$ in the intersection trees constructed above. In Theorem 3.3, we give a description of this term for the case of $K=\mathbb{Q}$, and we obtain Corollary 4.7 through it. If all the irreducible components of $X^{\text {sing }}$ are of degree strictly greater than 1 , we can use the estimates introduced in $\S 3.4$ to get a better estimate of

$$
\sum_{\xi \in S(X ; B)} \mu_{\xi}(X)\left(\mu_{\xi}(X)-1\right)^{n-s-1}
$$

than that given in Corollary 4.7. The reason is that we have a better estimate of

$$
\max _{Z \in \mathcal{Z}_{t}}\left\{\frac{N(Z ; B)}{\operatorname{deg}(Z)}\right\}
$$

than that given in Theorem 3.3 in this case, where $Z \in \mathcal{Z}_{t}$ is defined same as in Theorem 4.5 .

Example 4.9. - Let $\delta \geqslant 3$ and $n \geqslant 2$ be two integers. Let

$$
Z \hookrightarrow \mathbb{P}_{\mathbb{Q}}^{n+2}=\operatorname{Proj}\left(\mathbb{Q}\left[X, Y, T_{0}, \ldots, T_{n}\right]\right)
$$

be the hypersurface defined by the homogeneous polynomial

$$
F\left(X, Y, T_{0}, \ldots, T_{n}\right)=Y^{\delta}+X f\left(T_{0}, T_{1}, \cdots, T_{n}\right),
$$

where $f\left(T_{0}, \ldots, T_{n}\right)$ is an irreducible homogeneous polynomial of degree $\delta-1$ which defines a smooth hypersurface in $\mathbb{P}_{K}^{n}$, noted by $Z^{\prime}$ this hypersurface. The polynomial $F\left(X, Y, T_{0}, \ldots, T_{n}\right)$ is irreducible. By [18, Exercise 2.4.1], we obtain that the hypersurface $Z$ is integral. By [3, Theorem 1, Corollary], for all integers $\delta \geqslant 3$ and $n \geqslant 2$, and for any $\epsilon>0$, the estimate

$$
N\left(Z^{\prime} ; B\right) \ll_{n, \delta, \epsilon} B^{n-1+\epsilon}, \quad B \geqslant 1
$$

holds uniformly for every smooth hypersurface $Z^{\prime}$.

Meanwhile, by Jacobian criterion (cf. [18, Theorem 4.2.19]), the singular locus of $X$ is defined by

$$
0=F\left(X, Y, T_{0}, \ldots, T_{n}\right)=\delta Y^{\delta-1}=f\left(T_{0}, \ldots, T_{n}\right)=X \frac{\partial f}{\partial T_{0}}=\cdots=X \frac{\partial f}{\partial T_{n}} .
$$

Because the hypersurface $Z^{\prime}$ is smooth over $\mathbb{Q}$, the polynomials $f, \frac{\partial f}{\partial T_{0}}, \ldots, \frac{\partial f}{\partial T_{n}}$ have no common non-zero solutions. Hence for each $\xi \in Z^{\operatorname{sing}}(\overline{\mathbb{Q}})$, the projective coordinate $\left[x: y: t_{0}: \cdots: t_{n}\right]$ of $\xi$ satisfies $x=y=0$ and $\left[t_{0}: \cdots: t_{n}\right] \in Z^{\prime}(\overline{\mathbb{Q}})$, and every singular points is of multiplicity 2 in $Z$. By definition, $Z^{\text {sing }}$ is of codimension 2 in $Z$, 
whose dimension is $n-1$. We consider the sum in Theorem 4.5 for this example, for arbitrary integers $\delta \geqslant 3, n \geqslant 2$, and for all $\epsilon>0$, the equality

$$
\sum_{\xi \in S(Z ; B)} \mu_{\xi}(X)\left(\mu_{\xi}(X)-1\right)^{2}=2 N\left(Z^{\prime} ; B\right), \quad B \geqslant 1
$$

is verified for all hypersurfaces defined by the method in (4.6), (4.7).

We follow the construction in $\S 4.2$, where the proper intersection of $Z, V\left(\frac{\partial F}{\partial X}\right)$ and $V\left(\frac{\partial F}{\partial Y}\right)$ generates the only root of the intersection tree, and it has no descendent. Then we apply Theorem 4.5 to this case directly, and we obtain the inequality

$$
\sum_{\xi \in S(Z ; B)} \mu_{\xi}(X)\left(\mu_{\xi}(X)-1\right)^{2} \leqslant \delta(\delta-1)^{2} \frac{N\left(Z^{\prime} ; B\right)}{\delta-1}=\delta(\delta-1) N\left(Z^{\prime} ; B\right), \quad B \geqslant 1 .
$$

This is an example which satisfies the upper bound given in Theorem 4.5, since $\delta \geqslant 3$.

By [3, Theorem 1, Corollary], the estimate

$$
\sum_{\xi \in S(Z ; B)} \mu_{\xi}(X)\left(\mu_{\xi}(X)-1\right)^{2}=2 N\left(Z^{\prime} ; B\right) \ll_{n, \delta, \epsilon} B^{n-1+\epsilon}, \quad B \geqslant 1
$$

holds uniformly for all hypersurfaces defined by the method in (4.6), (4.7) and all $\epsilon>0$. In this case, the above estimate gives a better dependance on $B$ than that given in Corollary 4.7. But in this estimate, we have no description of the order of $\delta$, since we cannot control the order of $\delta$ in the above estimate to the extent of our current knowledge.

Similar to [17, Conjecture 5.13], we propose the following conjecture.

Conjecture 4.10. - Let $K$ be a number field, and $\delta \geqslant 1, d \geqslant 1, s \geqslant 0$ be three integers. The estimate

$$
\sum_{\xi \in S(X ; B)} \mu_{\xi}(X)\left(\mu_{\xi}(X)-1\right)^{d-s} \ll_{n, K} \delta^{d-s+1} B^{s+1}
$$

holds uniformly for all reduced pure dimensional closed subschemes $X$ of $\mathbb{P}_{K}^{n}$ of dimension $d$ and degree $\delta$, whose dimension of singular locus is $s$, where $S(X ; B)$ is defined in (3.2).

\section{References}

[1] T. D. Browning \& D. R. Heath-Brown - "Counting rational points on hypersurfaces", Journal für die Reine und Angewandte Mathematik 584 (2005), p. 83-115.

[2] _ "The density of rational points on non-singular hypersurfaces. I", The Bulletin of the London Mathematical Society 38 (2006), no. 3, p. 401-410.

[3] _ " "The density of rational points on non-singular hypersurfaces. II", Proceedings of the London Mathematical Society. Third Series 93 (2006), no. 2, p. 273-303, With an appendix by J. M. Starr.

[4] T. D. Browning, D. R. Heath-Brown \& P. Salberger - "Counting rational points on algebraic varieties", Duke Mathematical Journal 132 (2006), no. 3, p. 545-578.

[5] T. D. Browning - Quantitative arithmetic of projective varieties, Progress in Mathematics, vol. 277, Birkhäuser Verlag, Basel, 2009. 
[6] H. Flenner, L. O'Carroll \& W. Vogel - Joins and intersections, Springer Monographs in Mathematics, Springer-Verlag, Berlin, 1999.

[7] W. Fulton - Algebraic curves. An introduction to algebraic geometry, W. A. Benjamin, Inc., New York-Amsterdam, 1969, Notes written with the collaboration of Richard Weiss, Mathematics Lecture Notes Series.

[8] _ Intersection theory, second ed., Ergebnisse der Mathematik und ihrer Grenzgebiete. 3. Folge. A Series of Modern Surveys in Mathematics [Results in Mathematics and Related Areas. 3rd Series. A Series of Modern Surveys in Mathematics], vol. 2, Springer-Verlag, Berlin, 1998.

[9] X. Gao - On Northcott's theorem, ProQuest LLC, Ann Arbor, MI, 1995, Thesis (Ph.D.)-University of Colorado at Boulder.

[10] A. Grothendieck - "Éléments de géométrie algébrique. II. Étude globale élémentaire de quelques classes de morphismes", Institut des Hautes Études Scientifiques. Publications Mathématiques (1961), no. 8, p. 222.

[11] Q. Guignard - "Counting algebraic points of bounded height on projective spaces", Journal of Number Theory 170 (2017), p. 103-141.

[12] R. Hartshorne - Algebraic geometry, Springer-Verlag, New York, 1977, Graduate Texts in Mathematics, No. 52.

[13] D. R. Heath-Brown - "The density of rational points on curves and surfaces", Annals of Mathematics. Second Series 155 (2002), no. 2, p. 553-595.

[14] M. Hindry \& J. H. Silverman - Diophantine geometry, an introduction, Graduate Texts in Mathematics, vol. 201, Springer-Verlag, New York, 2000.

[15] G. Laumon - "Degré de la variété duale d'une hypersurface à singularités isolées", Bulletin de la Société Mathématique de France 104 (1976), no. 1, p. 51-63.

[16] C. Le Rudulier - "Points algébriques de hauteur bornée sur la droite projective", $J$. Théor. Nombres Bordeaux 26 (2014), no. 3, p. 789-813.

[17] C. LiU - "Comptage des multiplicités dans une hypersurface sur un corps fini", arxiv: 1606. 09337 (2016).

[18] Q. LiU - Algebraic geometry and arithmetic curves, Oxford Graduate Texts in Mathematics, vol. 6, Oxford University Press, Oxford, 2002, Translated from the French by Reinie Erné, Oxford Science Publications.

[19] D. Masser \& J. D. VAaler - "Counting algebraic numbers with large height. II", Transactions of the American Mathematical Society 359 (2007), no. 1, p. 427-445.

[20] J. Neukirch - Algebraic number theory, Grundlehren der Mathematischen Wissenschaften [Fundamental Principles of Mathematical Sciences], vol. 322, SpringerVerlag, Berlin, 1999, Translated from the 1992 German original and with a note by Norbert Schappacher, With a foreword by G. Harder.

[21] P. Salberger - "On the density of rational and integral points on algebraic varieties", Journal für die Reine und Angewandte Mathematik 606 (2007), p. 123-147.

[22] S. H. Schanuel - "Heights in number fields", Bulletin de la Société Mathématique de France 107 (1979), no. 4, p. 433-449.

[23] W. M. Sснмidt - "Northcott's theorem on heights. II. The quadratic case", Acta Arithmetica 70 (1995), no. 4, p. 343-375.

[24] J.-P. SERre - Local algebra, Springer Monographs in Mathematics, Springer-Verlag, Berlin, 2000, Translated from the French by CheeWhye Chin and revised by the author. 
September 22, 2018

Hao Wen, Department of Mathematical Sciences, Tsinghua University, Beijing 100084, P. R. China E-mail : wen-h10@mails.tsinghua.edu.cn

Chunhui Liu, Department of Mathematics, Faculty of Science, Kyoto University, Kyoto 6068502, Japan - E-mail : chunhui.liu@math.kyoto-u.ac.jp 